\title{
Phosphonate controls sudden oak death pathogen for up to 2 years
}

by Matteo Garbelotto and Douglas J. Schmidt

Since its emergence in the late 1990s, sudden oak death has killed mature oak trees and tanoaks in 14 California counties. Treatment options are now available to safeguard these trees from infection by Phytophthora ramorum, the aggressive and exotic pathogen responsible for sudden oak death. We provide an update on current knowledge regarding this emergent disease in California, and present results from three controlled experiments of two chemical treatments to manage the disease in oaks and tanoaks. Phosphonate treatments, legally registered in California to control sudden oak death, were effective in slowing both infection and growth rates for at least 18 months. Conversely, an alternative method consisting of an azomite soil amendment and bark lime wash was always ineffective, and did not reduce either growth or infection rates.

Sudden oak death (SOD), the $S$ emergent forest disease plaguing four oak species and tanoaks in 14 California counties, is caused by the introduced, nonnative pathogen Phytophthora ramorum (Garbelotto et al. 2001; Rizzo et al. 2002). Trees are infected by the pathogen through intact bark, and death is caused by lesions girdling the tree's cambium and partly occluding its vascular system. The time from infection to death can range from a few months to a few years, depending on environmental conditions and genetics of the host and pathogen. Eventually, the majority of girdled trees experience sudden browning of the entire crown without thinning or the loss of foliage, giving the disease its name (Rizzo and Garbelotto 2003).

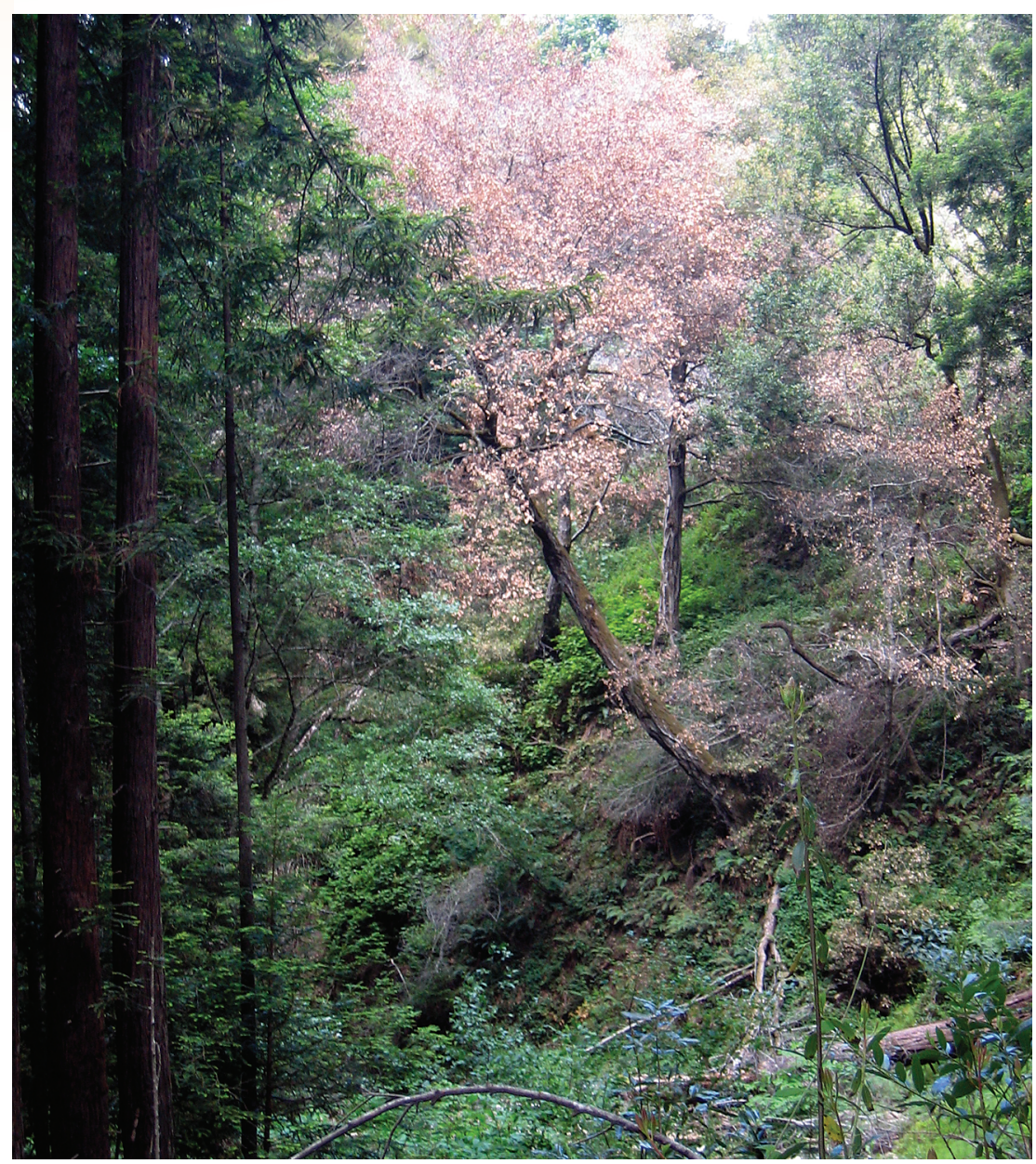

Oaks and tanoaks in 14 California counties are plagued by sudden oak death, including, above, tanoaks in the Big Sur region. The tree disease is caused by the exotic pathogen Phytophthora ramorum.

\section{Sudden oak death epidemiology}

Host plants. Although the disease kills large numbers of oaks and tanoaks, $P$. ramorum is a broad generalist capable of infecting more than 100 plant species and ferns (USDA APHIS 2007). On most hosts other than oaks and tanoaks, infection results in leaf blotches and branch diebacks that only occasionally kill infected plants. However, infected non-oak hosts are responsible for the disease's spread. In California woodlands, for instance, the surfaces of California bay laurel leaves and tanoak twigs produce large numbers of infectious airborne P. ramorum propagules, called sporangia. In commercial nurseries and European wildland settings, camellias and rho- dodendrons appear to play a key epidemiological role in spreading $P$. ramorum. Oaks are defined as "dead-end" hosts because they are seldom if ever infectious to other plant species (Garbelotto et al. 2003).

Infection propagules. While most Phytophthoras found in temperate forests are soilborne and waterborne (Erwin and Ribeiro 1996), P. ramorum is characterized by a predominant aerial phase (Davidson et al. 2005). During the rainy season, infectious sporangia are produced on the leaf surfaces or twigs of infectious hosts, and are released to the environment in wind or rainstorms. Once sporangia land on an area of a suitable host that is covered by a film of water, infection occurs by the release of motile zoospores 

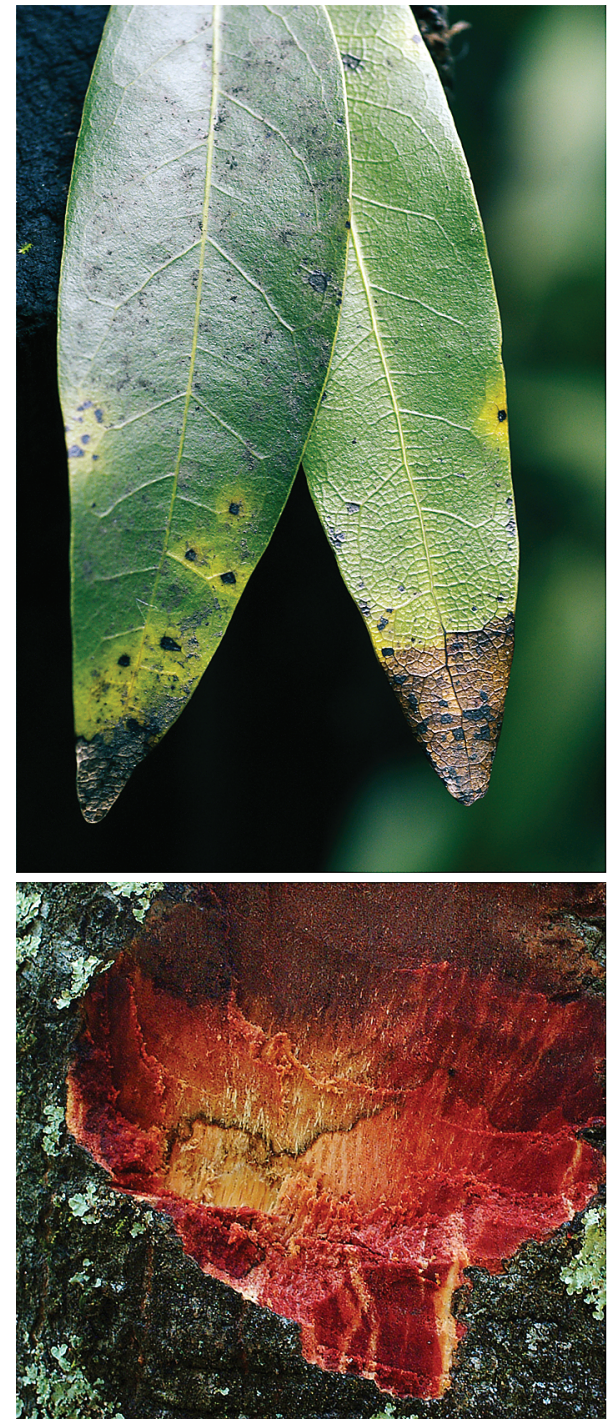

(see photo, page 12) that swim until they can penetrate the host tissue. Epidemiological observations suggest that infection success may be highest when temperatures are warm. In unfavorable dry conditions, and at any time on some plant hosts, $P$. ramorum produces chlamydospores, which are survival or resting propagules characterized by a thick protective outer layer.

Dispersal. Large numbers of

P. ramorum sporangia, zoospores and chlamydospores eventually end up either in the soil or in waterways. While the epidemiological role of pathogenbearing soil and water has not been documented, their potential roles range from being short-distance infection sources (such as by infested soil splashing onto the main bole of a tree), to being agents of long-distance dispersal (such as by infested waterways

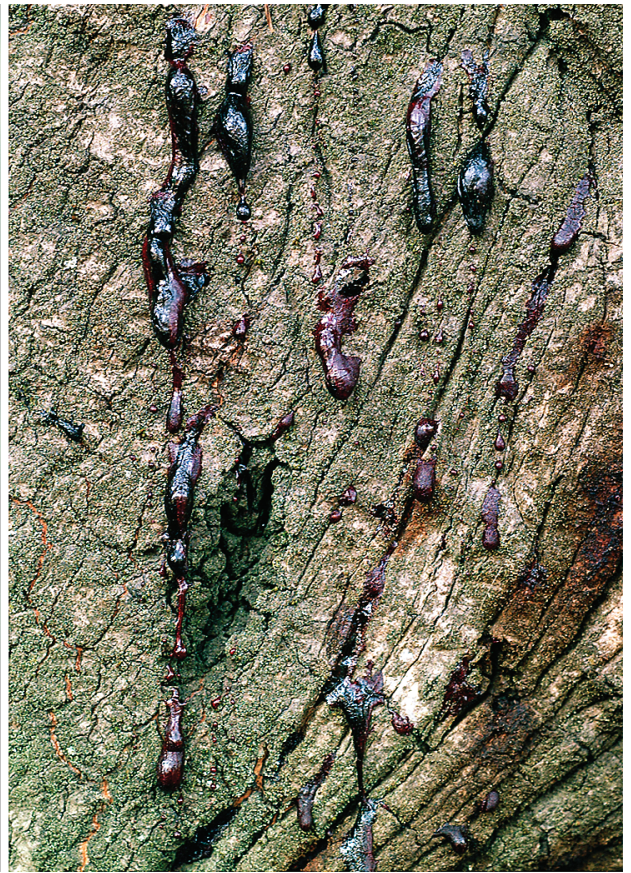

Top left, California bay laurel leaves typically become infected with $P$. ramorum on the tips of their leaves, where water and dew collect; bay leaves are believed to play a significant role in the disease's spread to oak trees. Top right, sudden oak death infections often produce weeping or bleeding cankers and may emit a thick, dark ooze from tree bark; this discharge is the tree's attempt to defend itself against infection. Left, in a mature coast live oak tree trunk, the black line shows the edge of the infection and marks the zone between living and dead woody tissue.

carrying the pathogen downstream for tens or hundreds of miles).

Although the pathogen's exact introduction date and its area of origin are not known, a recent genetic analysis reconstructed the history of sudden oak death in California and elucidated the pathogen's dispersal ability (Mascheretti et al. 2008). P. ramorum appears to have escaped the plant nursery environment in Santa Cruz and Marin counties, probably in the 1980s. Humans further enhanced dispersal of the pathogen in the 1990s around the San Francisco Bay Area, presumably by moving infected plants, as indicated by the fact that identical pathogen genotypes were found at distances up to 100 miles, a range that exceeds the natural dispersal ability of this microorganism. Natural spread of the pathogen may be occurring very efficiently at distances up to 200 yards, and presumably in the presence of strong winds, up to 3 miles. Natural movement over 6 to 7 miles appears to be extremely rare.

These inferences of the pathogen's spread are congruent with rates of spread for symptoms. Individual forest patches a few hundred yards long can be colonized by P. ramorum within one season, while movement of the pathogen between forest patches 0.5 to 3 miles apart can also occur at least once during the course of a rainy spring (Meentemeyer et al. 2004; Prospero et al. 2007). Long-distance spread is not well understood, but may rely on rare long hauls of propagules in strong winds or be linked to human movement.

Oaks and tanoaks. Although any plant subject to high levels of infection by $P$. ramorum may perish, oaks and tanoaks normally die in large numbers. The average mortality of oaks and tanoaks varies between $10 \%$ and $40 \%$, but at the local level mortality levels over $50 \%$ for adult oaks and up to $100 \%$ for adult tanoaks have been recorded (Davidson et al. 2005; Maloney et at. 2005). Besides their ecological importance, oaks and tanoaks enhance residential landscapes and can significantly improve property values. As backyard trees, oaks and tanoaks have become part of the cultural and social heritage of California. As keystone species of coastal ecosystems, indigenous people revere them as sacred trees.

\section{Chemical treatments tested}

Phosphonate. Chemical phosphonate treatments are registered to control sudden oak death in oaks and tanoaks, and are most effective if administered preventively before trees are infected (Garbelotto et al. 2007). Phosphonates are environmentally friendly, narrowspectrum fungicides that can be applied either by injection in the outer wood or topically on the bark. The topical treatments involve adding the organosilicate surfactant Pentrabark, which does not play any direct role in disease control but allows phosphonate to be absorbed through the bark.

Phosphonates are not toxic to animals, including fish and invertebrates, and do not affect beneficial microorganisms. Instead, these simple derivatives of phosphorous acid enhance the 

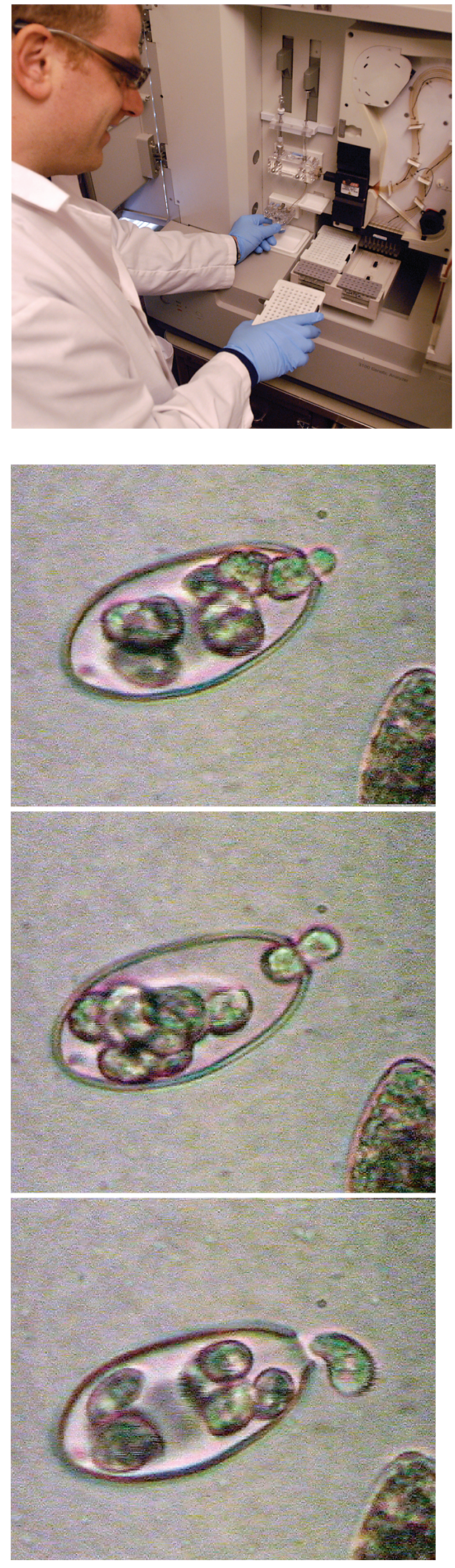

Above, three stages of motile, swimming $P$. ramorum zoospores are released from infectious and airborne sporangia, which are normally produced by $P$. ramorum on the surface of California bay laurel leaves during the spring rainy season.
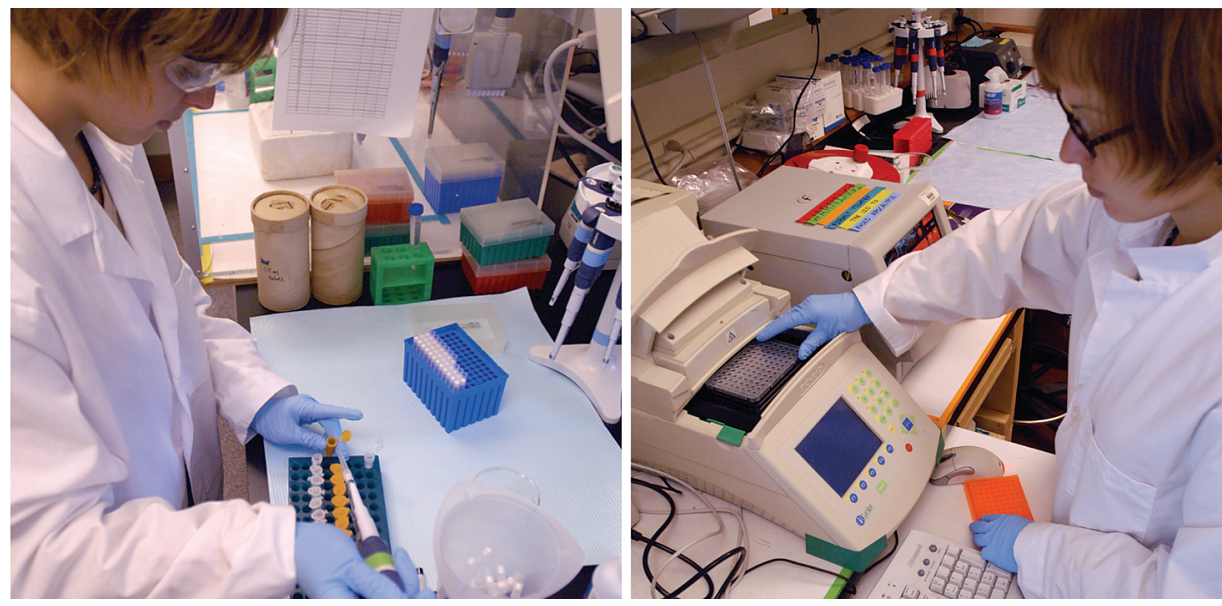

$\Delta$ Left, UC Berkeley postdoctoral researcher Noah Rosensweig uses a DNA sequencer to analyze DNA from $P$. ramorum; middle, UC Berkeley staff research associate Lydia Baker extracts DNA from $P$. ramorum and, right, uses real-time PCR to analyze it.

production of secondary metabolites that act as antibiotics (Garbelotto et al. 2008), helping treated plants to fend off microbial infections, including those by P. ramorum. The efficacy of phosphonate treatments has already been proven (Garbelotto et al. 2007), but such treatments were developed only relatively recently and no information is available on the duration of their efficacy.

Azomite and lime wash. Several unregistered and unproven treatments are also available to purportedly control sudden oak death in oaks and tanoaks. In particular, one alternative treatment involves amending soil with azomite and using a bark lime wash (see www. suddenoaklife.com). Azomite is an organic trace mineral added to soil as a fertilizer, while lime washes are meant to supply calcium to the plant. Supporters of this approach claim that these treatments decrease soil acidity in affected areas and provide nutrients to infected trees, thereby strengthening their defense mechanisms. The legality of this treatment is questionable because it lacks an official registration in California or elsewhere in the United States; furthermore, the absence of published scientific experiments makes it difficult to evaluate not only its merit and efficacy, but also the presence of potential side effects.

In this study, we present data from the first controlled experiment investigating the longevity of phosphonate treatments. We also present a novel way to test treatment efficacy on adult trees in the field without inoculating their main stems, and describe results of an experiment comparing the efficacy of the registered phosphonate treatment with that of the alternative azomite and lime wash treatment.

\section{Longevity and efficacy studies}

Potted oak experiment. We used 45 potted coast live oak trees that were 6 years old, 7 to 10 feet tall, and grown in 15-gallon pots filled with a 50:50 mix of sand and fir mulch. Trees were kept onsite in a lathe house at UC Berkeley for 2 months before the experiment started. On Nov. 30, 2005, 15 trees were injected with Agrifos (Agrichem, Queensland, Australia), the only phosphonate currently registered to treat sudden oak death in California. Another 15 trees were treated with a bark application of Agrifos and the organosilicate surfactant Pentrabark (Quest Products, Louisburg, KS), which is also registered for sudden oak death in California. The surfactant helps to even application of the phosphonate and enhances its adhesion on the tree trunk, so that the phosphonate can be progressively absorbed into the cambium. The remaining 15 trees were untreated controls.

Injections were applied with Chemjet injectors (Chemjet Trading, Queensland, Australia) each containing 10 milliliters of the phosphonate formulation at a concentration of 217 milligrams per milliliter. For the bark applications, we sprayed a solution of 310 milligrams per milliliter Agrifos and 1\% Pentrabark on a 1-yardhigh band of the tree main stems, until the bark surface was completely wet. 
Approximately 150 milliliters of solution per square meter of bark was used for this application.

Pr102, a widespread P. ramorum genotype (Ivors et al. 2004), was inoculated under bark of the main stem of treated and positive control saplings as described in Garbelotto et al. (2007). As a negative control, each tree was also mock-inoculated by placing a plug of sterile agar on the opposite side of the stem and 5 inches higher than the pathogen inoculation point. The mock inoculation allows researchers to determine the size of lesions caused by wounding during the inoculation process, in the absence of the pathogen. For each treatment and control, five trees were inoculated 6,12 and 18 months post-treatment.

Six weeks after each inoculation, we removed the outer bark and measured the extent of the necrotic lesions. To confirm that lesions were caused by $P$. ramorum, at least four isolations were taken by excising the margins of each visible lesion and plating them on a Phytophthora-semiselective culture media called PARP (Erwin and Ribeiro 1996). For each sapling, we calculated the cumulative linear lesion size by adding the maximum linear extent of the lesion along the stem to the maximum lesion extent across the stem's circumference. The data was then logtransformed and analyzed by ANOVA; multiple comparisons were performed with $t$-tests at alpha $=0.05$. This experiment was repeated for phosphonate injections, but the results were analogous to the first experiment and are not shown.

Field study. We selected 15 mature coast live oak trees in Novato, Calif., whose trunks with diameters at breast height (DBH) ranged from 24 to 48 inches and averaged 31 inches. On Feb. 14,2005 , five trees were treated as recommended by the manufacturer with phosphonate and organosilicate surfactant on the bark (approximately 1.35 gallons of tank mix per tree; mix composed of Agrifos at 0.07 ounce per gallon and $1 \%$ Pentrabark). Another five trees were treated as recommended by the distributor by adding azomite (143 pounds per tree) to the top organic layer of soil from the tree trunk to the canopy drip line, and applying lime wash to the bark from
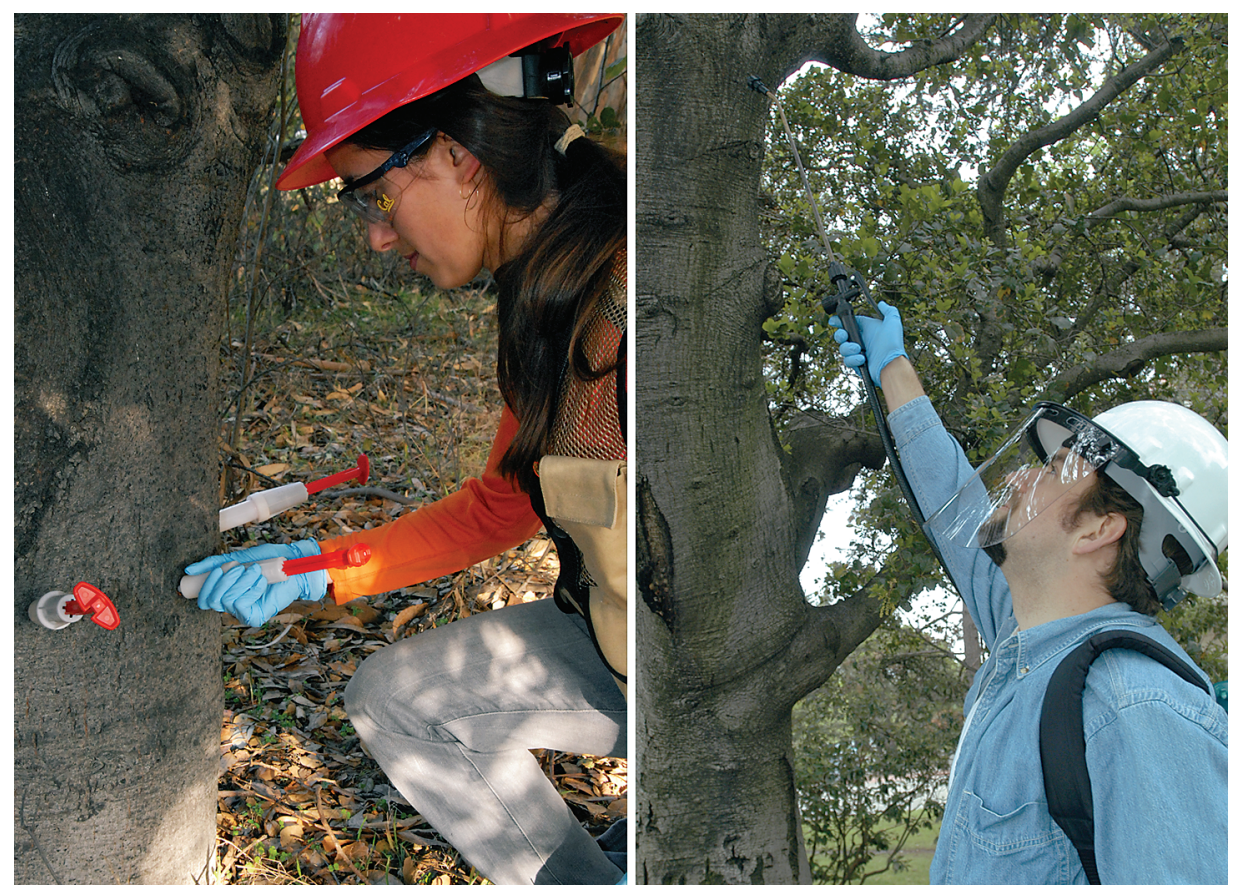

UC Berkeley staff research associate Ellen Crocker uses spring-loaded mechanical syringes to inject phosphonate compounds into a Marin County coast live oak.

the root collar to 5 to 6 feet up the trunk at approximately 2 gallons per tree. The remaining five trees were left untreated to serve as controls.

The efficacy of these two chemical treatments was tested at 6,12 and 18 months post-treatment by inoculating 10 cut branches per tree with P. ramorum as described in Dodd et al. (2005). Terminal branches (0.8 to 1.2 millimeters diameter) were cut, immediately placed in water and transported to the UC Berkeley greenhouse. Cuttings were transferred to fresh water and kept in a mist chamber. The bark was slit with a scalpel, and a plug of agar recently colonized by $P$. ramorum isolate Pr102 was placed under the bark. Mock control inoculations were performed on one branch per tree by placing a plug of sterile agar under the bark. All inoculations were wrapped with parafilm (Pechiney Plastic Packaging, Menasha, WI) and aluminum foil.

Three weeks after inoculation, we removed the outer bark and measured the extent of necrotic lesions. To confirm that these lesions were caused by P. ramorum, at least two isolations were taken from the edges of each visible lesion and plated on PARP. For each cutting, the extent of the lesion upward toward the tip of the branch was added to the extent of the lesion downward to-
UC Berkeley staff research associate Brett Voss applies a topical phosphonate spray to a coast live oak in Alameda County to study the treatment's effectiveness.

ward the branch base. Data was then logtransformed and analyzed by ANOVA; multiple comparisons were performed by $t$-tests with alpha set at 0.05 .

Efficacy testing. For most plant diseases caused by Phytophthora spp., there is a good correlation between the size of lesions caused by under-bark inoculations and susceptibility of the individual plant (Huberli et al. 2002). By wounding the inoculation site, underbark inoculations bypass the infection process and thus test the rate of pathogen spread rather than that of infection. Because it could be argued that some treatments effectively prevent infection but do not necessarily slow disease progression, we designed a way to directly test the efficacy of treatments in preventing infection by $P$. ramorum.

To circumvent the limitations of under-bark inoculations, we used a more realistic inoculation test on 40 potted oaks like those used in the first experiment. Before treatment, the bark was gently scraped at the selected inoculation points to ease direct infection by zoospores. We treated 10 trees with a bark application of phosphonate and organosilicate surfactant (Agrifos and Pentrabark) and 10 with the azomite soil amendment and bark lime wash; 20 were left untreated. Fourteen days later, the 20 treated trees and 10 


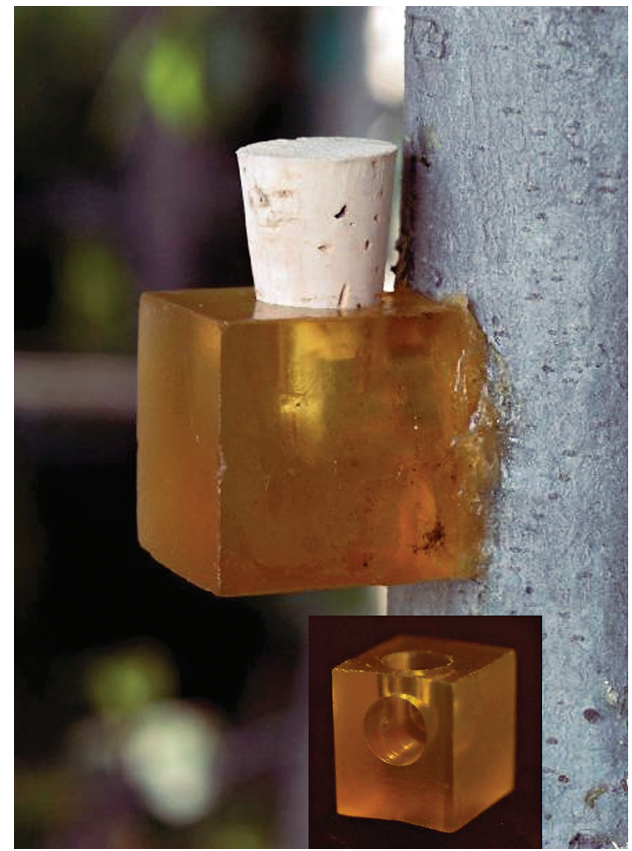

A plastic device developed at UC Berkeley is used to artificially infect trees with zoospores of $P$. ramorum.

of the untreated trees were inoculated with zoospores of Pr102 isolates. The other 10 untreated control trees were mock-inoculated using water without zoospores.

To produce $P$. ramorum zoospores, ten 1-square-centimeter plugs were cut from the margins of 2-week-old colonies and floated on sterile deionized water in 100-millimeter-diameter Petri dishes. The plates were incubated in the dark at room temperature for 72 hours (Erwin and Ribeiro 1996). At the end of the incubation period, the water used to flood each of the 10 plates was poured into a plastic bottle with a 250-milliliter-wide mouth (Nalgene, Rochester, NY). To obtain additional zoospore solution, all plates were rinsed a second time with

\section{Phosphonate treatments} reduce both colonization and infection rates of the sudden oak death pathogen.

10 milliliters of sterile deionized water, and second rinses were added to the first ones in the same bottles. The bottle cap was then secured without tightening it, to allow for air flow. We induced sporangia to release zoospores by placing the bottle on ice for 30 minutes, followed by 1 hour at room temperature.

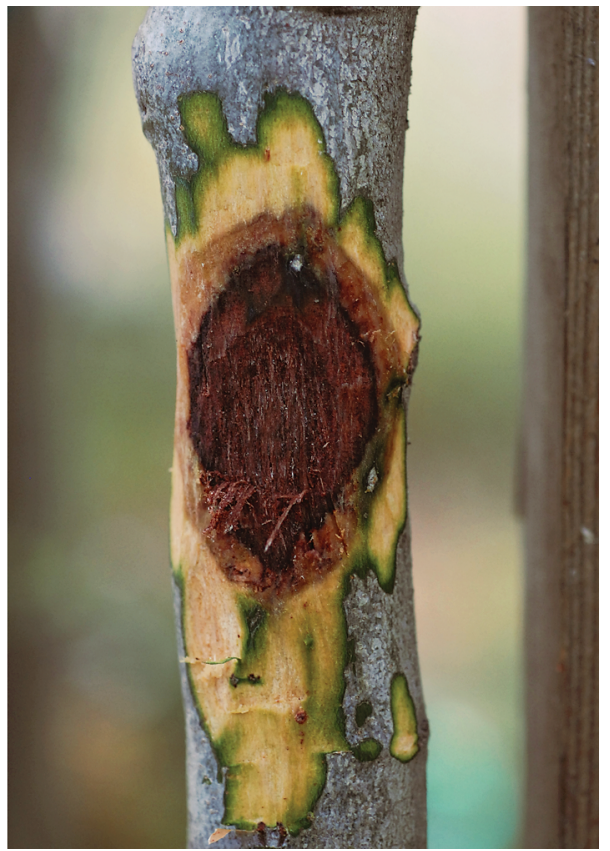

The dark lesion in this Alameda County coast live oak may continue to grow until it girdles and eventually kills the tree.

In order to visually verify that zoospore release had occurred, zoospore counts were performed using a hemacytometer (Hausser Scientific, PA).

Once zoospores were produced and their concentration adjusted to $1 \times 10^{4}$ per milliliter, 1.5 milliliters of zoospore suspension was added to an inoculation block placed around the inoculation point. The blocks, made out of polyurethane resin, had concave surfaces that conformed to the round bark surface. Each inoculation block had two openings: one in the concave side to allow delivery of zoospores to the inoculation point, and another on top to allow the operator to place the desired aliquot of zoospore suspension in the block. Grafting wax was used to ensure a perfect seal between the inoculation block and tree surface. Blocks were left for 24 hours with the upper opening corked. After the blocks were removed, the inoculation points were entirely wrapped with parafilm and foil.

Six weeks after inoculation, we removed the outer bark and measured the extent of necrotic lesions. To confirm that these lesions were caused by $P$. ramorum, at least four isolations were taken from the edges of each visible lesion and plated on PARP. For each sapling, we calculated the cumulative linear lesion size by adding the maxi- mum linear extent of the lesion along the stem to the maximum lesion extent across the stem's circumference. The data was then log-transformed and analyzed by ANOVA; multiple comparisons were performed by $t$-tests with alpha set at 0.05 .

\section{Treatment comparisons}

Phosphonate and potted oaks. Compared to the untreated trees, cumulative lesions were significantly smaller in the potted trees treated with phosphonate injections and bark applications of phosphonate and organosilicate surfactant (Agrifos and Pentrabark) for each post-treatment dataset $(\mathrm{df}=2 ; 25<F<$ $76.3 ; 0.001<P<0.0001$ ), as indicated by ANOVA performed separately on the 6-, 12- and 18-month datasets (fig. 1). Treatment efficacy was also detected 1 and 3 months post-treatment (data not shown). In all cases, P. ramorum was isolated at least once from each inoculated tree, independent of treatment, confirming that the lesions visible in the phloem were caused by the inoculated pathogen. Negative mock inoculations never caused any lesions beyond the wound-induced one, and were excluded from the analysis.

Field study. The branch cuttings that we placed in water and kept in a mist chamber survived well during the length of each inoculation trial. $P$. ramorum was reisolated at least once from each inoculated branch, confirming that lesions visible in the phloem were caused by the inoculated pathogen. There was never a significant difference between the size of lesions in untreated trees and in those treated with the azomite soil amendment and bark lime wash, as indicated by ANOVAs performed on each individual dataset of inoculations at 6,12 and 18 months after treatment (fig. 2). Conversely, the average lesion size in tree branches treated with bark applications of phosphonate and organosilicate surfactant was always significantly smaller than those in untreated tree branches $(\mathrm{df}=2 ; 56<F<$ 79.4; $P<0.0001)$. Phosphonate efficacy was also detected 1 and 3 months posttreatment (data not shown).

Efficacy testing. Potted trees were successfully infected by zoospore suspensions applied through the inocula- 


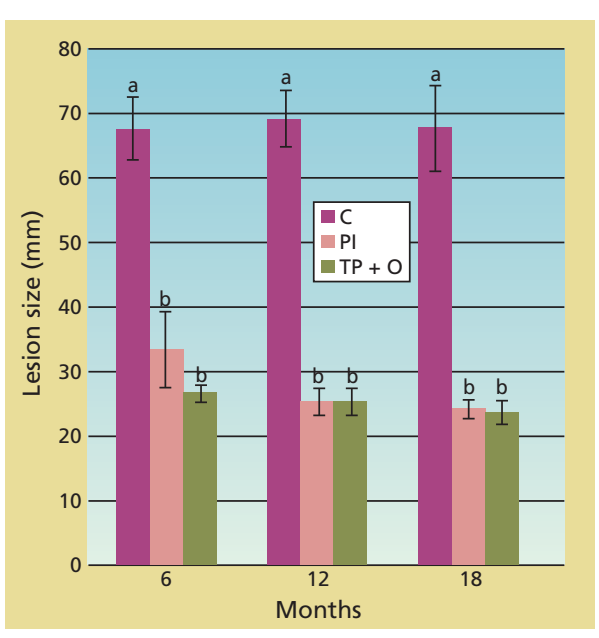

Fig. 1. Cumulative linear size of lesions (along and across stem) caused by under-bark inoculation of Phytophthora ramorum onto the main stem of potted trees at 6,12 and 18 months after treatment. Treatments included injections with phosphonate (PI), topical bark application of phosphonate and organosilicate surfactant $(T P+O)$ and untreated controls (C). Average lesion size and standard error in wounded but uninoculated negative controls were $\mathbf{1 6 . 6}$ and $\mathbf{0 . 6}$ millimeters, respectively.

tion blocks. Lesion sizes in trees treated with the azomite soil amendment and bark lime wash were not statistically different from those in untreated trees (fig. 3). In contrast, lesions in trees whose bark was treated with a topical application of phosphonate and organosilicate surfactant were both significantly smaller than those of untreated trees $(\mathrm{df}=2 ; F=37.6 ; P<0.001)$ and significantly larger than those of the mockinoculated trees.

\section{Recommendations for SOD treatment}

Introduced plant diseases represent one of the most serious threats to endemic plant communities in California and can lead to the decimation or even extinction of affected plant hosts (Wingfield et al. 2001). The high levels of mortality caused by sudden oak death among some oak species and tanoaks are a vivid example of the intensity and scale of damage that can be caused by an introduced microorganism. Genetic analyses confirm the exotic nature of the sudden oak death pathogen (Ivors et al. 2006; Mascheretti et al. 2008), intensifying the need for approaches aimed at both slowing down the epidemic and protecting tree species lethally affected by $P$. ramorum.

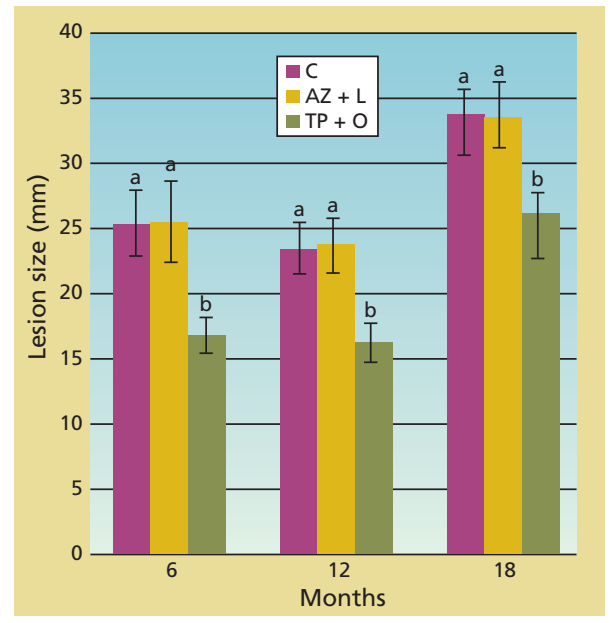

Fig. 2. Total linear size of lesions along stems of branch cuttings caused by under-bark inoculation of Phytophthora ramorum at 6, 12 and 18 months from treatment of parent trees from which cuttings came. Treatments included topical bark application of phosphonate and an organosilicate surfactant (TP + O), azomite soil amendment and bark lime wash $(A Z+L)$ and untreated control (C). Average lesion sizes (standard error) in wounded but uninoculated negative controls were $14(0.3), 13(0.2)$ and 18 (0.3) millimeters, respectively, for the 6-, 12and 18-month trials.

Phosphonates have long been used in agricultural situations, including avocado orchards in California to control other Phytophthora species (Guest et al. 1995), as well as in natural ecosystems, including the Jarrah forests of Western Australia (Hardy et al. 2001). Phosphonates are fungicides targeting a small but important group of plant pathogens called oomycetes. The action of phosphonates is often dual, with a direct contact effect augmented by an increased defense response in the treated plant, which may include a range of physiological changes from cell-wall thickening to the increased production of antimicrobial secondary metabolites (Guest and Grant 1991). However, $P$. ramorum is not particularly susceptible to direct contact with phosphonate (Garbelotto et al. 2008), and most of this treatment's efficacy hinges on the augmented production of defensive secondary metabolites.

While registered in California to treat sudden oak death in oaks and tanoaks, phosphonate treatments are not necessary for blue oak, valley oak, Oregon oak or interior live oak, all of which have never been reported as infected by P. ramorum. Because sudden oak death is an emergent disease with

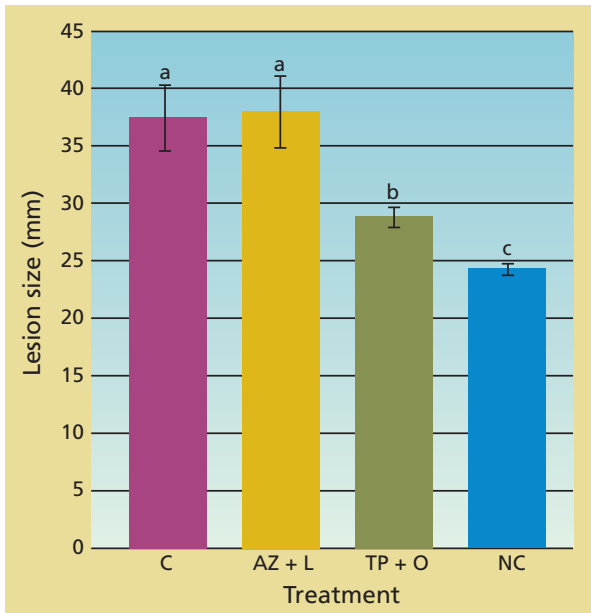

Fig. 3. Cumulative linear size of lesions (along and across stem) caused by applying zoospores of Phytophthora ramorum onto main stem of potted trees $\mathbf{4 2}$ days after treatment. Treatments included topical bark application of phosphonate and organosilicate surfactant (TP +0$)$, azomite soil amendment and bark lime wash (AZ $+\mathrm{L}$ ), untreated controls (C) and negative control in which bark was gently scraped but no zoospores were applied (NC).

only a recent history in California, the longevity of efficacy for registered treatments is unknown, although controlled studies have shown that treatments are most effective if administered preventively before trees are infected (Garbelotto et al. 2007).

Phosphonate treatments have been effective for 2 to 6 years in other Phytophthora species, depending on the host species (Hardy et al. 2001). Our first potted tree experiment and the field experiment indicated that phosphonate treatments had a significant effect at 6,12 and 18 months postapplication; future testing will deter-

\section{The azomite and lime bark}

\section{wash showed no efficacy} whatsoever.

mine whether they are effective for longer. Because most $P$. ramorum infections occur in late winter and spring, 18 months of coverage will adequately protect trees for 2 years. However, in order to obtain the highest level of control using phosphonate treatments, a recommended schedule of preventive applications for oaks and tanoaks includes two applications the first year to ensure 

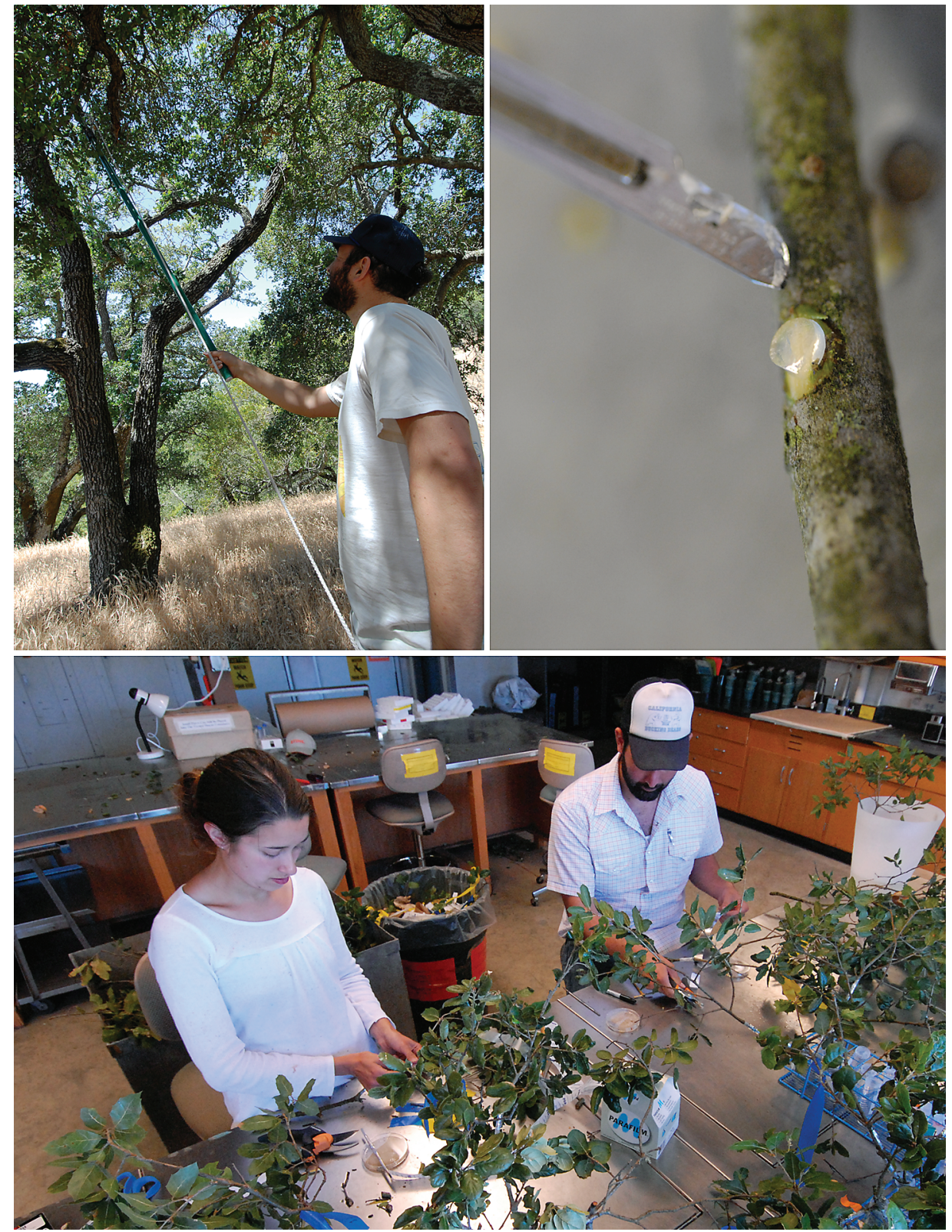

Top left, UC Berkeley staff research associate Alex Lundquist uses a pole pruner to collect branches of a Marin County coast live oak. Top right, a small plug of agar is used to infect the branches with $P$. ramorum. Above, UC Berkeley staff research associates Ellen Crocker and Lundquist inoculate oak branches in the laboratory. This study method allows researchers to safely infect oak wood with $P$. ramorum in the laboratory and test various sudden oak death treatments.

full coverage (one in late fall and one in early spring), followed by one fall treatment every year. Fall treatments are recommended because they allow the plant to develop a full defensive response before infection spreads in the warm and rainy spring.

The choice of phosphonate application method (injection or bark spray) is to some extent a personal one. Injections release the totality of the product inside the tree without the organosilicate surfactant, presum- ably achieving efficacy faster; but specialized equipment and training are needed to drill multiple holes in the tree, and a certain number of injections may fail, especially in gnarly trees with abundant decay pockets. Although a single injection was applied to our potted trees, larger trees require multiple injections, approximately one for every 6 inches (15 centimeters) of tree circumference. Holes are drilled at a slight downward angle through the bark and cambium into the outer three to six growth rings of the wood. Absorption of the chemical appears to be fastest on warm sunny days, from the late morning to midafternoon.

Bark topical applications are rapid and easy; however, post-application efficacy takes longer, the organosilicate surfactant must be used in conjunction with phosphonate, the chemicals can disperse into the environment, and all types of foliage including moss are at risk of phytotoxicity upon contact with the chemical. It is best to mix the phosphonate and organosilicate surfactant on treatment day. Applications can be done with any kind of applicator or sprayer. The lower 10 feet of trunk must be thoroughly wetted, possibly including some of the lower main branches in the canopy.

The field experiment - in which adult trees were treated but efficacy was tested on cut branches rather than on the entire tree - was successful. This approach may provide a new tool to study treatment options for sudden oak death and other forest diseases without killing plants or using dangerous inoculum in the field. P. ramorum grew significantly slower in trees inoculated with phosphonates at 6,12 and 18 months after treatment, while the pathogen's growth rate with the azomite and lime wash treatment was always the same as that of untreated controls. We found that the phosphonate topical treatment was effective up to 18 months from application, while the azomite and lime bark wash showed no efficacy whatsoever at any of the three time periods tested.

The second potted tree experiment was designed to directly assess the efficacy of treatments on infection rather than colonization rates of the pathogen. The results indicated that the phosphonate topical application significantly reduced the size of infection lesions caused by zoospores, but the azomite and lime wash treatment had no effect. Besides this lack of efficacy, we found that the sheer amount of material needed to treat each tree with azomite and lime wash was cumbersome and aesthetically unpleasant due to the large amounts of azomite on the ground around treated trees. Although this treatment is being touted as a holistic fertilizing approach aimed at en- 
